\title{
ASPECTOS ECONOMICOS DE LAS SOCIEDADES SOCIALISTAS
}

\author{
Benjamín Bastida i Vilà \\ (Universitat de Barcelona)
}

No es fácil abarcar en los límites de un artículo las características económicas de las sociedades socialistas, ya que, aunque vistas desde Occidente podrían dar la impresión de un bloque monolítico, un análisis un poco más pormenorizado descubrirá diferencias sustanciales entre ellas. Los procesos de reforma, iniciados en todas ellas hace más de veinte años, han recorrido caminos distintos; lo han hecho, sobre todo, a ritmos diferentes, y en algún caso, como Polonia, han estallado en forma de graves crisis sociopolíticas.

Sin embargo, a pesar de la diversidad, la Unión Soviética sigue cumpliendo el papel de «hermano mayor», de centro y punto de referencia de los intentos de reforma o, en todo caso, de «Gran Hermano» cuya aprobación es necesaria. Fijémonos en que muchas veces, al referirse a la reforma húngara (la más avanzada en la puesta en práctica del llamado «socialismo de mercado» o «mercado guiado»), los especialistas emplean la expresión «el experimento húngaro». Es como decir que lo llevado a término en los seis pequeños países europeos del CAEM tiene un carácter de «provisionalidad», de importancia sólo relativa.

Esta calificación es indudablemente injusta, pues la experiencia llevada a cabo, por ejemplo, en Hungría tiene un valor objetivo en sí misma y puede servir de modelo para otros paises del CAEM de dimensión semejante, con mayores probabilidades de éxito que la trasposición estalinista de finales de los cuarenta del modelo de la Unión Soviética. Sin embargo, dicha califfcación traduce una realidad del sistema en su conjunto, tal como hoy por hoy existe: es en la URSS donde hay que tomar el pulso a la economía de 
planificación central «realmente existente» para diagnosticar su capacidad o incapacidad de reforma.

Con las salvedades y justificaciones precedentes puede resultar comprensible que, al abordar la exposición de las características económicas de las sociedades socialistas, centremos nuestra atención en los rasgos sobresalientes de la Unión Soviética, por más que, respecto a dicho país, la impresión dominante - siempre desde la perspectiva y las noticias llegadas a Occidente- no sea la de cambios radicales sino, en todo caso, de procesos de «adaptación y mejora» -como dice el discurso oficial- muy lentos.

\section{LOS ANALISIS DE LA REFORMA ECONOMICA SOVIETICA}

El rasgo dominante de la economía soviética al comenzar la segunda mitad de los ochenta -inicio del XII Plan Quinquenal- es la persistencia de las instituciones y los mecanismos de funcionamiento que podrían englobarse bajo la denominación genérica de «planificación estalinista» de la economía nacional, sólo superficialmente modificados por las sucesivas reformas de 1965 y 1979, como hitos principales.

Desde esta perspectiva también podría hablarse, tal vez con mayor propiedad, de que la característica de la economía soviética en la fase actual es el fracaso de los sucesivos intentos de reforma iniciados en 1965. Los cambios, introducidos sólo a medias, no han resuelto los graves problemas para los que fueron propiciados y, por el contrario, han generado un sinfín de ambigüedades en los procesos económicos y en las relaciones entre los diversos escalones de la burocracia de la producción: Centro Planificador, Oficina de Precios, Organismos de Suministro de Materias Primas y Bienes de Equipo, Ministerios, directores de empresas...

Se ha escrito abundantemente sobre este tema a lo largo de los últimos diez o quince años, desde perspectivas diversas pero con un notable acuerdo sobre el fondo de las cuestiones.

En una especie de resumen cronológico podrían distinguirse tres etapas en estos estudios. La primera consiste en el análisis de las reformas introducidas en 1965 y en la valoración de los resultados, sumamente contradictorios como he dicho, que se producen en la economía soviética. Los últimos análisis de esta fase llegan a la Reforma Económica de 1979.'

En un segundo momento el centro alrededor del cual giran los estudios es el tema de la «crisis» en las economías del Este. La pregunta surge, en

1. Sin pretensión de exhaustividad, me atrevería a recomendar como vision panorámica de estos estudios: 
parte, como derivación del estudio de la crisis en las economías occidentales, pero también por razones internas de las economías centralizadas: la más llamativa es el estallido de la crisis polaca, pero asimismo se analizan las repercusiones de la crisis mundial sobre el bloque socialista, los aumentos de la deuda exterior de esos países, las consecuencias de los «ciclos de inversión» características de las economías centralizadas, etc. Podría decirse que en el examen de los datos hay gran consenso entre los especialistas, pero no así en el diagnóstico de la existencia o no de «crisis socialista». Los puntos de desacuerdo comienzan, naturalmente, en el mismo concepto de crisis aplicable - ¿qué es «crisis económica socialista», supuesto que el concepto deriva de análisis aplicados a la economía capitalista? Otro punto de divergencia es el juicio acerca de los fenómenos antiguos, casi consustanciales a las economías centralizadas - ¿por qué hablar abora de crisis si ya desde siempre han existido las deficiencias denunciadas? ¿Ha llegado el sistema a sus límites? La recuperación de los años 1982-1983 parece negar una afirmación rotunda. El tercer enfoque, en el que se aprecian diferencias entre los especialistas, consiste en la importancia que unos y otros dan a los aspectos políticos y sociales para caracterizar la crisis. Desde este punto de vista - por la concomitancia de tensiones económicas, sociales y políticasestán todos de acuerdo en que en Polonia el sistema ha experimentado realmente una crisis. Finalmente, la predicción sobre el futuro de estas economías y, más en concreto, de la economía soviética, vuelve a ser un punto de discusión entre los especialistas.

Una excelente referencia bibliográfica sobre la crisis en las economías socialistas es el conjunto de estudios, coordinados por la profesora M. Lavigne y el profesor W. Andreff, aparecido hace un año bajo el título La Réalité Socialiste: Crise, Adaptation, Progrès. ${ }^{2}$ El libro cosecha el fruto de dos

Campbell, R. W., Soviet-Type Economics: Performance and evolution, Londres, Macmillan, 1974.

Fallenbuchl, Z. B. (ed.), «Economic Development in the Soviet Union and Eastern Europe», vols. I y II (Selección de ponencias del Congreso de Bahnf), Nueva York, Praeger, 1975-1976.

Lavigne, M., Les Economies Socialistes Soviétique et Européennes, París, Colin, 1970 (1." edic.); última edición en 1983.

Nove, A., The Soviet Economy System, Londres, Allen and Unwin, 1977.

- La segunda parte de mi tesis doctoral aborda también estas cuestiones (Bastida, B., El sistema impositivo en la Unión Soviética y su contribución a la eficiencia productiva, Universidad de Barcelona, Facultad de Ciencias Económicas y Empresariales, 1979). Un resumen actualizado se publicó en «Hacienda Pública Española», núm. 66.

Específicamente sobre la Reforma Económica de 1979, Bastida, B., La Reforma Económica de 1979 en la URSS, «Inprecor», enero de 1980; «La URSS, Plan Quinquenal y Reforma», en Libro del Año 1981, Barcelona, Salvat, 1982. 
años de sesiones de Seminario del Grupo de Investigación sobre la teoría de la economía socialista, ubicado en el Centre d'Economie Internationale des Pays Socialistes de la Universidad de París I (Pantheon-Sorbonne). En el libro queda recogida esta diversidad de enfoques a la hora de examinar los graves desajustes actuales de las economías centralizadas. ${ }^{3}$ Varios de los autores presentaron en el III World Congress on Soviet and East European Studies celebrado en Washington, en noviembre de 1985, un «panel» bajo el significativo título: "What about Economic Crisis in the CMEA Countries?», cuyas ponencias se publicarán en francés en la Révue d'Etudes Comparatives Est-Ouest en el tercer número de 1986.

La etapa más reciente de los estudios sobre la economía de las sociedades socialistas, superpuesta parcialmente en el tiempo a la que acabamos de describir, destaca los problemas más acuciantes a los que éstas se enfrentan en el final del siglo $\mathrm{xx}$, o si se quiere más en concreto, aquéllos que son calificados como más apremiantes por sus respectivas burocracias en el poder. Dentro de la orientación general de las reformas necesarias para pasar de un modelo de crecimiento extensivo a un modelo de crecimiento intensivo (según la formulación oficial de dichos países, ya tópica en la literatura sobre el tema), parecen sobresalir unos cuantos objetivos prioritarios.

El modelo de crecimiento extensivo, por recordarlo en pocas palabras, consiste en la adición de factores al proceso de producción (más mano de obra, más recursos naturales, más equipos e instalaciones) para alcanzar así tasas elevadas de aumento de la renta nacional. El modelo de crecimiento intensivo, por contraposición, consiste en lograr los aumentos gracias al aprovechamiento «más intenso» de una cantidad de factores productivos, cantidad que, por unas $u$ otras circunstancias, no puede ya incrementarse. La utilización intensiva de los factores de producción exige cambios técnicos, organizativos e institucionales en el modo de gestión y producción que son precisamente los que la reforma trata de introducir con poco éxito, según hemos comentado antes.

En este contexto de fracaso de la reforma y de necesidad de «reforma de la reforma», se inicia en los últimos años, sobre todo en la Unión Soviética, una estrategia de prioridades urgentes y de experiencias sectoriales en el terreno económico. El análisis de esta estrategia está centrando la aten-

2. Lavigne, M., y Andreff. W. (coord.), La Realité Socialiste: Crise, Adaptation, Progrès, Economica, París, 1985.

3. Otras referencias bibliográficas que podrían servir de complementos serían:

Drach, M., La crise dans les pays de l'Est, París, La Découverte, 1984.

Richet, X. (coord.), Crise à l'Est?, París, Presses Universitaires de France, 1984. 1985.

Dyker, D. A., The Future of the Soviet Planning System, Londres, Croom Helm, 
ción de los especialistas (varias ponencias del último Congreso Mundial ya citado se referían al tema), pero hay que decir que se trata, todavía, de estudios relativamente novedosos.

Por ello, más que repetir o intentar un resumen de temas conocidos, puede ofrecer un mayor interés el referirse al período de estos dos últimos años y a las perspectivas que se abren para la Unión Soviética, de aquí a fin de siglo, con la llegada de Mikhail Gorbachev al poder. Tras la larga decrepitud de Breznev y los interregnos de Andropov y Chernenko, un líder desacostumbradamente joven (para los hábitos de la URSS) y técnicamente bien preparado (ésa es la imagen que se ha difundido y que él mismo ha cuidado en Occidente) ocupa la cúpula soviética.

\section{LA MODERNIZACION DE GORBACHEV}

Parece cierto, en efecto, que Gorbachev ha percibido la necesidad y es partidario decidido de una modernización a fondo del funcionamiento económico de la Unión Soviética. Su lenguaje político en este sentido - no siempre, pero sí con más frecuencia que sus predecesores- es directo y concreto. Abonaría esta afirmación, por ejemplo, el hecho de haber devuelto a la comisión correspondiente del Gosplan el proyecto del Plan Quinquenal que debía aprobarse en el Congreso; el mismo hecho de haber cesado al que era presidente del Comité del Gosplan desde hacía muchos años, N. Babaikov; el haber creado una comisión específica - y haberle dado orientaciones muy directas - para estudiar el tema de la «aceleración del progreso científico y técnico»; el haber suprimido y reagrupado en un único Ministerio los varios Ministerios agrícolas; las declaraciones de aprobación de la reforma húngara, etc.

A mitad de camino de los «mil días» de Gobierno, es preciso, sin embargo, confesar que en el aspecto económico contamos con más gestos y declaraciones que con cambios sustanciales en el terreno de los hechos. Vale la pena, con todo, analizar tanto unos como otros para diseñar las líneas de la reforma que Gorbachev se propone acometer.

En mi opinión, la estrategia de modernización de Gorbachev se está dibujando alrededor de tres líneas maestras: el avance tecnológico, la disciplina en el trabajo y la lucha contra la corrupción. No son, evidentemente, inventos reformadores del nuevo líder soviético, sino que ya de antiguo se habían considerado elementos necesarios. La insistencia en ellos no equivale a renunciar a las otras pautas de la reforma de 1965 y de 1979 . Pero parece evidente que Gorbachev ha puesto un énfasis específico en estos tres pun- 
tos. Este énfasis, la insistencia en su control, al darles un carácter decisivo, es lo nuevo de la estrategia.

La renovación tecnológica de la economía soviética - «la aceleración del progreso científico y técnico» en el lenguaje oficial- parece configurarse como el pilar principal y absolutamente urgente de la reforma de Gorbachev. La elección de esta estrategia parece acertada. Constituye ya un tópico entre los especialistas la consideración de que uno de los defectos principales de la economía de la URSS es su incapacidad para incorporar de una forma fluida el progreso tecnológico, lo que da como resultado la falta de calidad y el escaso nivel técnico de muchos de sus productos.

Las causas de este atraso tecnológico no hay que buscarlas en la falta de investigación básica o aplicada. Tomando como base la misma estadística oficial soviética, hemos podido comprobar, ${ }^{4}$ a través de una serie de indicadores seleccionados, que los niveles de investigación básica y aplicada son elevados y se desarollan en el tiempo; que los esfuerzos y las políticas de introducción de los resultados de esa investigación en la industria mantienen unos ritmos muy aceptables. Sin embargo, los resultados económicos obtenidos en la aplicación industrial son cada vez peores. En definitiva, el proceso de Investigación y Desarrollo (R. \& D.) consume cada vez más «inputs» y produce «outputs» menores.

¿Qué significado hay que atribuir a esta conclusión que se basa, subrayémoslo, en la estadística oficial soviética? Parece indudable que el atraso tecnológico y la relativa incapacidad de progreso se deben a causas organizativas o institucionales, a defectos en la gestión, precisamente, del avance tecnológico. El análisis estadístico revela el divorcio existente entre las empresas y los organismos de investigación y de planificación. La pretendida y aparente corrección de los laboratorios de investigación y de los «laboratorios» económicos de los planificadores choca con la resistencia de las empresas al cambio tecnológico. ¿Cuál es la razón de esta resistencia? La empresa soviética está sometida a una tensión contradictoria si es «escogida» para introducir procesos nuevos. Por una parte, ha recibido un plan anual de producción que responde teóricamente a sus posibilidades o, en definitiva, a lo que la empresa ha realizado en años anteriores más un cierto porcentaje de crecimiento de la producción. Debe cumplir dicho plan inexorablemente. Por otra, como empresa potencialmente innovadora, está sujeta a la imposición, venida de arriba, de introducir mejoras tecnológicas en sus procesos y en sus productos. Como es bien conocido, la innovación requiere

4. Bastida, B., y Virgili, M. T., "Crisis and Technology: The U.S.S.R.», ponencia presentada en el III World Congress on Soviet and East European Studies (Washington, noviembre de 1985). Versión francesa en «Revue d'Études Comparatives EstOuest», vol. 17, ת. 3 . 
normalmente un período de adaptación, un suministro en buenas condiciones y en los plazos adecuados, una formación del personal para el manejo provechoso de dichas innovaciones. Hay un riesgo evidente de que el cumplimiento del plan de producción se vea seriamente comprometido.

El «manager» soviético, sometido a una jerarquía burocratizada, no se siente dispuesto a asumir tales riesgos. De algún modo, jugando con las palabras, podría decirse que no tiene más remedio que defenderse a sí mismo y a la empresa que dirige contra las innovaciones impuestas administrativamente para poder cumplir las imposiciones conservadoras del Plan.

Esta situación, que se refiere al sistema económico en su conjunto, se corresponde con el análisis del estado de la tecnología en las diversas ramas industriales. Las industrias que podríamos denominar «clásicas», como, por ejemplo, la siderurgia, están tecnológicamente avanzadas a nivel mundial. En cambio, las ramas que corresponderían a la alta tecnología -electrónica, informática, etc.- presentan un atraso muy considerable. ${ }^{5}$

Capítulo aparte merece la industria de la defensa y la de investigación del espacio, donde las aplicaciones de alta tecnología son absolutamente necesarias y en las que la Unión Soviética se configura como segunda potencia mundial (en algún aspecto y durante determinados períodos, incluso como primera). La constatación de este hecho nos remite, de nuevo, a los rasgos institucionales de la economía soviética. Demuestra, por una parte, que no hay un atraso tecnológico «alarmante» en los niveles de investigación básica y aplicada. En segundo lugar, la peculiar y privilegiada situación de la industria de la defensa y del espacio posibilita que los resultados de aquellos experimentos de laboratorio se trasladen con éxito a la producción industrial del sector de la defensa.

Aunque las empresas del sector militar oponen, como todas, cierta resistencia al cambio tecnológico - de ahí las características del desarrollo del armamento soviético: perfeccionamiento de modelos antiguos más que introducción de sistemas nuevos, acumulación más que precisión, etc.-, reciben de su cliente, el Ministerio de Defensa, las especificaciones técnicas del producto y están sometidas a controles rigurosos de calidad y plazos de entrega. Paralelamente, gozan de prioridad absoluta para conseguir a tiempo los suministros en la cantidad y la calidad requeridas. Abraham Becker, sovietólogo norteamericano, comentaba en una conferencia impartida en

5. Ésta es la conclusión general que puede extraerse de los valiosos estudios realizados por el Centre for Russian and East European Studies de la Universidad de Birmingham.

Amman, R., y Cooper, J., The Tecbnological Level of Soviet Industry, New Haven, Yale Univ. Press, 1972.

Amman, R., y Cooper, J. Industrial Innovation in the Soviet Union, New Haven. 
Barcelona en junio de 1985, que la «peculiaridad tecnológica» del sector de defensa soviético se debe a que dicho sector está sometido a la competencia del mercado internacional, puesto que el cliente conoce los productos de dicho mercado a tenor de lo cual formula y revela sus necesidades, lo que no sucede en el sector de la industria civil. No se me escapa que la ingeniosa observación del profesor Becker parece ir en el sentido de contraponer las virtudes del mercado a los defectos inherentes a la planificación. Para no caer en simplificaciones ni embarcarnos en una discusión que supera los propósitos de este artículo, habría que añadir que el cliente es no sólo «solvente», sino capaz de proporcionar a la empresa productora una situación privilegiada en el ámbito de la economía planificada. ${ }^{6}$

En tercer lugar, la diferencia de niveles tecnológicos entre el sector militar y el sector civil en la economía soviética es una muestra de la falta de difusión de tecnología entre ambos sectores; rasgo asimismo sustantivo de la URSS, atribuible no sólo a los condicionantes de la planificación soviética ya referidos, sino posiblemente también a la forma de garantizar el «secreto de Estado» que rodea a todo lo que tenga relación con la Defensa. ${ }^{\text {? }}$

\section{EL RETO TECNOLOGICO}

El «reto tecnológico» de Gorbachev pretende modificar este panorama. descrito a grandes rasgos. ¿Cuáles son las armas escogidas para afrontar este reto? De forma sucinta podríamos clasificarlas según las áreas geográficas en que se proyectan: 1) En la propia URSS; 2) en el CAEM, y 3) en las relaciones con el Occidente industrializado.

1. Las estrategias definidas por M. Gorbachev para acelerar el proceso científico y técnico mediante modificaciones internas del proceso económico soviético guardan estrecha relación, naturalmente, con las propuestas generales de renovación del sistema de gestión y producción. De algún modo, podría decirse que la estrategia tecnológica aspira a polarizar y robustecer dichas propuestas generales. Éstas, como ya dijimos anteriormente, consisten. en un reforzamiento de la disciplina en el trabajo y en la lucha contra la corrupción (además, naturalmente, de la innovación tecnológica en sí misma).

6. Para una explicación más completa del carácter prioritario del sector Defensa en la economía soviética consúltese:

Virgili, M. T., «El Gasto en Defensa en la Unión Soviética», tesis doctoral, Uni-versidad de Barcelona, 1985, cap. VI.

7. Virgili, M. T., op. cit., cap. VI (esp. 6. 2.3.). 
El reforzamiento de la disciplina en el trabajo y su correlato, la lucha contra la corrupción, se concretaron, en los primeros meses del mandato del nuevo secretario general, en la lucha contra el alcohol, causante de una cierta proporción de absentismo laboral, y en la destitución pública, seguida de la imposición de severas penas, de algunos cargos significativos.

Ambos elementos, al confluir con la estrategia de innovación tecnológica, amplían su significado, al menos en términos retóricos de proclamación de objetivos y denuncia de defectos. La «disciplina en el trabajo» se convierte en «necesidad de participación activa y consciente de las masas», buscando los cauces adecuados para que desarrollen su potencial creativo. ${ }^{8} \mathrm{La}$ «lucha contra la corrupción» se extiende a denuncias muy concretas, no ya de corrupción propiamente dicha, sino de incompetencia o rutina en determinados Ministerios o Direcciones Generales.

En el terreno concreto de la ciencia y la tecnología, el documento más comprensivo y actual es el Informe presentado al XXVII Congreso del PCUS. ${ }^{9}$ Lo que parece más llamativo es la lucidez con que se denuncian las deficiencias del proceso de Investigación y Desarrollo. No hay paliativos para esas deficiencias. Sin embargo, la otra cara de la moneda, la de las propuestas, aparece muy difuminada: principios generales formulados ya otras veces, voluntad y llamamiento muy acentuados para provocar un cambio de actitud ante «lo nuevo», y algunas orientaciones (todavía no desarrolladas en normativas concretas) que podríamos considerar como relativamente originales con respecto a discursos de líderes anteriores.

Entre estas orientaciones cabe distinguir las que se refieren al trabajo científico, las que se refieren a las decisiones de inversión y las que van dirigidas a superar el divorcio entre investigación-experimentación y difusión masiva de la tecnología en el proceso productivo.

Las primeras pretenden, como era de esperar, estimular el trabajo creativo de los científicos, luchando contra el departamentalismo y el exceso de burocracia: establecimiento de incentivos materiales y de promoción para los investigadores, con cierta independencia de su lugar en el escalafón; constitución de una sección especial dentro de la Academia de Ciencias de la URSS dedicada a la informática y la cibernética; mejor aprovechamiento de la Universidad -investigadores, docentes y estudiantes - para la creación y la difusión de la tecnología. (Se propone, entre otras cosas, que los estudiantes, ya desde los primeros cursos, se familiaricen con la investigación y establezcan contactos con la práctica de las empresas productoras.)

8. Parece que han tenido que ver mucho con la formulación de estas propuestas los informes críticos de la economista de Novosibirsk, Tatiana Zalavskaia.

9. Informe Político del Comité Central del PCUS al XXVII Congreso, presentado por el secretario general, M. Gorbachev, el 25 de febrero de 1986. 
Dentro del segundo grupo - decisiones de inversión-, hay que destacar la toma de postura en favor de la reconstrucción y la modernización de las instalaciones ya existentes, frente a lo que venía siendo más habitual en la economía soviética: inversiones dedicadas a proyectos nuevos, a ampliaciones «extensivas» de la capacidad productiva, muchos de los cuales sólo llegaban a acabarse después de plazos larguísimos o bien, en el mejor de los casos, llevaban a la coexistencia de instalaciones obsoletas con otras relativamente modernas, en situaciones de muy dudosa rentabilidad. El giro de la estrategia era reclamado por los economistas soviéticos desde antes del advenimiento de Gorbachev y fue tomando cuerpo con su llegada al poder. Si efectivamente es llevado a la práctica, modificará sustancialmente el panorama industrial de la Unión Soviética. En este mismo grupo de orientaciones económicas se sitúa el criterio de elección entre inversiones alternativas del nivel tecnológico y la capacidad para provocar «saltos tecnológicos» contenida en las inversiones propuestas.

$\mathrm{El}$ divorcio entre ciencia y producción trata de superarse mediante la ampliación y el perfeccionamiento de experiencias anteriores, en especial la de los llamados «complejos tecnocientíficos intersectoriales». Se trata de un tipo de organización en la que se relacionan permanentemente los institutos de investigación, las organizaciones académicas y de confección de diseños y proyectos, y las plantas industriales piloto. Sin embargo, el mismo secretario general reconoce que, en este punto, el éxito no ha coronado la política diseñada: se ha procedido con una lentitud inadmisible $\mathrm{y}$, traduciendo literalmente, «muchos institutos no dejan de ser una continuación del aparato de los Ministerios, oficiando con frecuencia de abogados de los intereses departamentales y empantanándose en la rutina y el papeleo». ${ }^{10}$

2. Las estrategias relacionadas con el avance tecnológico que implican al CAEM se han plasmado en lo que ha venido denominándose el plan Eureka de los países del Este, es decir, el Programa Integral de progreso científico y técnico del CAEM hasta el año 2000, aprobado en la última Conferencia Económica Cumbre de los países miembros. En síntesis, se trata de sustituir las relaciones principalmente comerciales por relaciones de especialización y cooperación productiva — división del trabajo socialista-, llegando a la creación de agrupaciones y complejos científico-productivos conjuntos. La pregunta inmediata es si bajo el término de «cooperación» no va la URSS a tratar de aprovechar su situación preponderante para exigir a los países del CAEM, en especial a los más industrializados, Alemania del Este y Checoslovaquia, una aportación sustancial a su proyectado

10. Informe del Comité Central al XXVII Congreso del PCUS (\& II, B, 1). 
proceso de modernización. Habrá que estar atento, por otra parte, a algún fenómeno interesante que ha comenzado a desarrollarse en el CAEM dentro de este Programa Integral, como la fabricación de elementos de informática en Bulgaria, país tradicionalmente agrícola. En todo caso, las áreas. prioritarias del Programa están en consonancia con una política de modernización tecnológica: electrónica, automatización, energía nuclear, nuevos materiales y biotecnologías.

3. Con respecto a los países occidentales, la estrategia definida contiene ciertas ambigüedades. Por una parte está el dato cierto de que la URSS y en general los países del Este han sido importadores de tecnología occidental, especialmente en el decenio 1970-1980 (más de una tercera parte de sus importaciones de Occidente). Posiblemente, en determinadas áreas de tecnología avanzada, estas importaciones serán todavía útiles y necesarias. Por ello se habla de no renunciar a las ventajas del comercio internacional. Pero al mismo tiempo, por parte de los líderes soviéticos, se manifiesta una cierta oposición a estas importaciones. En el texto conocido. como «Orientaciones fundamentales del desarrollo económico y social de la URSS en 1986-1990 y hasta el año 2000"," Nikolai Rizhkov (nombramiento de confianza de Gorbachev) atribuye precisamente al «ansia de adquirir técnica y tecnología en el extranjero que se ha apoderado de numerosos dirigentes», el desánimo y la consiguiente falta de resultados de los equipos autóctonos de diseño de tecnología. Personalmente opino que las razones para esta postura relativamente contraria a la importación de tecnología occidental tienen un origen doble: uno es, en efecto, el que señala el presidente del Consejo de Ministros de la URSS, pero otro puede estar en las recientes dificultades económicas para obtener divisas fuertes, como derivación de la caída de los precios internacionales del crudo, y en la voluntad de escapar a una dependencia tecnológica de Occidente que podría crear algunas dificultades para el propio desarrollo en casos de embargo de tecnología, a través de la COCOM, por ejemplo.

\section{¿UN NUEVO ROSTRO DE LA SOCIEDAD SOVIETICA?}

Si estas estrategias pueden llevarse a término - junto con el resto de reformas económicas propuestas por Gorbachev, que constituyen casi una

11. Informe del presidente del Consejo de Ministros de la URSS al XXVII Congreso del PCUS (3 de marzo de 1986). 
condición para el cumplimiento de la renovación tecnológica-, los efectos se harán notar no tan sólo en la tasa de crecimiento de la Renta Nacional, sino en la vida cotidiana de los ciudadanos soviéticos. Piénsese, por ejemplo, que una de las áreas prioritarias de la introducción de nuevas tecnologías es la informática: las propuestas no contemplan simplemente la fabricación y la introducción de medios informáticos en los organismos del Plan Central, de los Ministerios y de las empresas, sino que, con buen criterio, se introduce la informática en la educación escolar y se proyecta la difusión de los ordenadores personales. En un país donde se cuenta, aunque sea sólo como anécdota, que la realización personal de fotocopias tropieza con dificultades, la divulgación de medios para el almacenaje y el tratamiento de la información representa cambios sustanciales de actitud.

De algún modo, es el mismo concepto de información el que habrá de cambiar. De una información como «bien exclusivo» de los gestores económicos que se encargan de su utilización y distribución controlada, a una información como «bien público» de libre acceso, o de acceso mucho más libre. Este cambio de concepción socava, sin duda, una de las raíces de los privilegios de los burócratas. Pero, al mismo tiempo, puede ofrecer a los gestores de la economía planificada una información menos adulterada sobre las necesidades y los recursos del sistema en su conjunto. Podría, en definitiva, contribuir a la supresión de la viciosa dialéctica «imposición administrativa - picaresca empresarial» y a la clarificación de esa especie de «mercado negro» de la información existente, hoy por hoy, en la Unión Soviética. No sé hasta qué punto los máximos dirigentes son conscientes de estas consecuencias: aunque los discursos y los textos señalan la necesidad de aplicar urgentemente la informática, no se descubre en ellos esta nueva concepción . de la información a la que me estaba refiriendo.

Otras consecuencias de la difusión de la tecnología en los procesos económicos se han comenzado ya a experimentar en la Unión Soviética o han sido anunciadas por sus dirigentes. Eufemísticamente, se habla de la «liberación de fuerza de trabajo» que provocarán la automatización y la robotización de las fábricas. De hecho, aunque por causas diferentes - la reorganización de los Ministerios agrícolas a la que antes me he referido-, un cierto paro ficcional, subsidiado naturalmente, ha aparecido en la URSS. En las fábricas, de todos modos y por el momento, el «reajuste de plantillas» no se realiza (fuera del casi histórico experimento de Shchekino); ${ }^{12}$ coexisten la maquinaria nueva y la obsoleta o, en todo caso, el reajuste de plantilla es puramente interno: los trabajadores «liberados» quedan como «reserva

12. E1 artículo más reciente sobre el experimento citado es el siguiente: Nort, $\mathrm{H}$., Sbchekino: Another Look, en «Soviet Studies», abril de 1986. : 
de mano de obra» en la nómina de la empresa para los momentos de agobio ante el necesario cumplimiento del plan - final de mes o momentos de control central. Así se esterilizan potenciales incrementos de productividad del trabajo. Es evidente la contradicción de situaciones como la descrita con un proceso de modernización industrial.

\section{CONCLUSIONES EN UN CONTEXTO ECONÓMICO DIFICIL}

Las circunstancias económicas mundiales (caída del precio internacional del crudo, de cuya exportación la URSS obtenía casi el $60 \%$ de sus divisas fuertes) e internas (catástrofe de Chernobil y revisión del programa de energía nuclear) hacen que el reto tecnológico y modernizador que debe afrontar Gorbachev se dibuje en un horizonte sombrío.

Por otra parte, la lectura de sus declaraciones y de los informes al XXVII Congreso, deja al estudioso observador de la realidad soviética con una sensación combinada de expectación y escepticismo. Textos semejantes se produjeron en tiempos de Krushchev y de Kosigin y en la misma propuesta de Reforma de 1979, en el último período de Breznev. Las reformas, sin embargo, acabaron siendo absorbidas por la inercia del sistema. "Inercia del sistema» puede parecer una expresión abstracta. Traduce, en realidad, el poder de conservación de una burocracia que si propone reformas económicas es, en última instancia, como intento de resolver sus luchas internas por parcelas de poder más amplias.

Desde este punto de vista, en las últimas propuestas, los perdedores parecen ser los Ministerios donde, según las manifestaciones de Gorbachev y su nuevo equipo, los procedimientos burocráticos se hallan más enquistados, en el peor sentido de la palabra.

La incógnita es si la reforma abre el paso a un proceso democratizador - como los textos insinúan a través de las llamadas al potencial creativo de las masas soviéticas- o si enmascara la toma del poder por una burocracia de carácter tecnocrático y mucho más joven que la que hasta ahora detentaba las decisiones últimas. En este segundo caso, el más probable a mi entender, las contradicciones de la economía y de la sociedad soviética dificilmente van a resolverse en un contexto económico internacional, por otra parte, plagado de obstáculos y cercos al desarrollo de sistemas alternativos al capitalismo de las empresas transnacionales.

12. 